\title{
A numerical study of vibration-induced instrument reading capability degradation in helicopter pilots
}

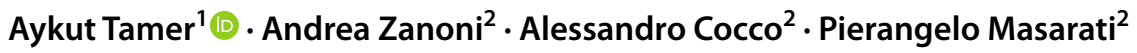

Received: 22 October 2020 / Revised: 11 March 2021 / Accepted: 2 April 2021 / Published online: 16 April 2021

(c) The Author(s) 2021

\begin{abstract}
Rotorcraft suffer from relatively high vibratory levels, due to exposure to significant vibratory load levels originating from rotors. As a result, pilots are typically exposed to vibrations, which have non-negligible consequences. Among those, one important issue is the degradation of instrument reading, which is a result of complex human-machine interaction. Both involuntary acceleration of the eyes as a result of biodynamics and vibration of the instrument panel contribute to a likely reduction in instrument reading capability, affecting flight safety. Therefore, being able to estimate the expected level of degradation in visual performance may give substantial benefits during vehicle design, allowing to make necessary adjustments while there is room for design changes or when retrofitting an existing aircraft to ensure the modifications do not adversely affect visual acuity and instrument reading ability. For this purpose, simulation is a very valuable tool as a proper model helps to understand the aircraft characteristics before conducting flight tests. This work presents the assessment of vibration-induced visual degradation of helicopter pilots under vibration exposure using a modular analysis environment. Core elements of the suggested analysis framework are an aeroelastic model of the helicopter, a model of the seat-cushion subsystem, a detailed multibody model of the human biodynamics, and a simplified model of ocular dynamics. These elements are combined into a comprehensive, fully coupled model. The contribution of each element to instrument reading degradation is examined, after defining an appropriate figure of merit that includes both eye and instrument panel vibration, in application to a numerical model representative of a medium-weight helicopter.
\end{abstract}

Keywords Rotorcraft aeroelasticity $\cdot$ Visual acuity $\cdot$ Pilot vibration exposure

\section{Introduction}

\subsection{Problem statement}

In helicopters, the forces and moments originating from the rotors, fuselage aerodynamics, engine and transmission create oscillatory loads. Once transmitted to the airframe, these time-dependent loads then propagate to the cockpit and the cabin. Time-dependent loads are intrinsic to the rotor dynamics, hence the resulting vibrations exist continuously yet with altering levels. As a result, the main source of discomfort in the helicopter cockpit and cabin is represented by sustained vibratory accelerations received by the occupants.

\footnotetext{
Aykut Tamer

a.tamer@imperial.ac.uk

Imperial College London, London, UK

2 Politecnico Di Milano, Milano, Italy
}

Additionally, other serious problems are also a direct result of vibrations, such as degraded pilot handling qualities [1, 2] and chronic pain frequently observed on the crew [3]. In addition to mentioned undesirable consequences, the vision acuity of pilots can also worsen as a result of vibrations in the cockpit $[4,5]$.

Considering a typical helicopter cockpit, the pilot rests on a seat in nearly vertical sitting posture. The pilot seat responds to cabin floor and structure vibrations, which are received by the human body. The involuntary motion of the human body transmits the received accelerations to the skull through the spine, and from the skull to the eyes. Therefore the image formed on the retina is not stationary. This aspect represents the subjective portion of the problem. Visual acuity degradation involves the subject's ability to see while being exposed to vibrations. Additionally, the cockpit displays also vibrate in response to excitation reaching them through a different load-path. This aspect represents the objective portion of the problem, namely the incorrect 
perception that the human eye-and central nervous system-receive from rapidly moving objects. Instrument reading ability degradation is a consequence of the combined subjective visual acuity degradation and objective vibration of the instruments. The differences between the amplitude and phase of the eye's and instrument panel's accelerations result in a relative vibratory motion between the eye and the visual displays. This causes degradation in visual acuity and instrument reading ability as the levels of vibration becomes severe. As a result, the pilot may start making reading errors and show a slower response to the changes in the displayed information. For example, Gardner [6] reports that during the development of the BAC TSR-2, a "reheat fuel-pump oscillation (...) was causing an airframe vibration [...] at a frequency very close to that of the human eyeball. As prototype XR219 climbed away on its second flight, this vibration happened, and 'Bee' (Roland Prosper 'Bee' Beaumont, the legendary RAF test pilot), to avoid blurred eye-sight, had to throttle out the port engine." Not a helicopter, but worth reporting, as it is indicative of what vibrations could cause in flight.

\subsection{Background}

The relative motion can be dominated by the vibration of the pilot eye or that of the display screens; however, it is likely that both contribute substantially [7]. The degradation is proportional to the amplitude of the vibration for both causes [8]. Being made of completely mechanical components from the source to the subjects, the vibration assessment of the display screens is relatively straightforward, once the mechanical properties of the system are known. On the other hand, analyzing the vibration response of the human body is more challenging, since it is characterized by intrinsic complexity, uncertainty, and individual variability. The biodynamic response of the human body depends not only on the input frequency but also on individual anthropometric and physiological parameters, and even on posture. This is especially critical in the case of involuntary motion of upper body parts, such as the head and eyes, where the identification of the dynamic response from measurements becomes more difficult and highly uncertain.

The available literature, for involuntary eye motion as a result of whole-body vibration, reports greater variability, across the relevant frequency range, as compared to pure mechanical response of the airframe structure. Nevertheless, some of the independent studies manifest similarities, thus making it possible to determine some trends. For example, in Ref. [9], Ohlbaum suggested that the eye response relative to the skull appears to increase at about $12 \mathrm{~Hz}$ and shows a peak in the vicinity of $18 \mathrm{~Hz}$. Otherwise, it was shown that the eye follows the skull with no noticeable amplification. Similarly, Ishitake [10] reported a maximum reduction of visual acuity at a frequency of $12.5 \mathrm{~Hz}$. Collins [11] noticed the significant contribution of human biodynamics on visual performance degradation above $10 \mathrm{~Hz}$, which compares favorably with the above-mentioned findings. In [12], $12 \mathrm{~Hz}$ is also indicated as the target frequency for the effect of vibration on visual acuity in a combined positive $G$ manoeuvre and sustained vibration . Lee and King [12] achieved a similar result and reported that the vibration of the eye as a result of whole-body vibration from a seat rises up to $12 \mathrm{~Hz}$ and then remains at the same order of magnitude. The frequency range for appreciable consequences of vibration on visual performance was observed up to $31.5 \mathrm{~Hz}$ by Lewis and Griffin [14]. The latter result is confirmed by Harazin et al. [15], who define an interval of maximal sensitivity between 12.5 and $31.5 \mathrm{~Hz}$, which is consistent with previously mentioned findings.

In addition to the amplitude and frequency of the input excitations, other factors can affect the display reading performance. Two critical parameters are the distance and orientation or the complexity of the displayed information [16]. Moreover, the brain interprets the visual input in a complex manner with the visual acuity and partially suppresses oscillations in the perceived image using cognitive corrections. Nakashima [7] reported that such an intervention likely occurs below the 1-2 $\mathrm{Hz}$ threshold, where the visual acuity can be corrected to some degree. However, the compensation is not efficient at higher frequencies, suggesting that the vibration of the image on the retina is perceived as it is. This aspect is believed to be helpful for the numerical prediction of visual acuity. Passive-i.e. involuntary - models, based on the mechanical response of human body and helicopter structures, can be expected to be relatively accurate for frequencies higher than $2 \mathrm{~Hz}$.

\subsection{State of the art and scope}

The most common solution to compensate visual degradation is to increase the size, contrast, and spacing of the displays or the graphical elements of the user interface (UI) [17]. This approach, however, limits the amount of information that can be submitted, especially in helicopters where the available area for displays is generally limited. Helicopter cockpit design solutions are therefore limited by the trade-off between the displays' size and vibration performance. In this context, the estimation of the attainable visual performance of a concept solution can offer a viable support, helping determining the relative contribution of design variables and choices while excluding non-feasible solutions as early as possible.

Comprehensive rotorcraft simulation aimed at biodynamic response is not straightforward and cannot be performed using a single tool without suffering from undue simplifications, especially when the vehicle and human 
dynamics are coupled. On the vehicle side, helicopters are composed of subcomponents, such as rotors, airframe, control system, etc., that have different characteristics, which requires a common framework that can efficiently incorporate different computational models. On the biodynamics side, the human body and the motion of the eye should be included to achieve realistic levels of eye acceleration. For this purpose, the authors enhanced a modular analysis platform developed over the past decade [18] to support the evaluation of visual instrument reading performance, culminating in the work of Ref. [19], which introduces a high-fidelity model and a figure of merit for visual acuity and instrument reading ability degradation. The relative significance of each contributing element can be evaluated.

The present work extends the idea initially proposed in Ref. [19] and supports it by providing additional results and further discussing the related findings. A more complete understanding is achieved in how to address pilot visual acuity and instrument reading ability degradation by addressing the relative contribution of pilot and panel vibration, the effects of uncertainty in eye response, and analyzing the consequences of the variability in thein strument panel acceleration..

To the authors' knowledge, a modular and versatile platform for visual performance evaluation of helicopter pilots subjected to the variety of vibratory loads that is typically experienced by the vehicle is not currently available to the rotorcraft community. Therefore, a novel contribution to the evaluation of pilot occupational health and to the improvement of flight safety can be achieved using the proposed approach. Moreover, the proposed visual vibration index can be further incorporated in a generalized vibration index, as the one proposed in [20], which accounts for all vibrationinduced aspects of discomfort for (rotorcraft) pilots.

\subsection{Organization}

This paper is composed of four Sections. Sect. 1 describes the proposed approach and illustrates how it can be used to evaluate visual performance. A high-fidelity analysis model is subsequently developed in Sect. 3, and its building blocks are explained. Then, the effect of significant parameters is investigated in Sect. 4. Finally, in Sect. 5 general conclusions are drawn from the presented results.

\section{Method}

In this section, a modeling environment suitable for formulating an overall high-fidelity model for rotorcraft aeromechanics and vibration is explained. A framework for the assessment of visual performance is formulated as well, and incorporated in the aeromechanical model.

\subsection{High-fidelity modeling environment}

In-flight evaluation of visual acuity and instrument reading ability degradation is almost impossible for safetyrelated reasons. Although simulator testing in principle is possible, it is nonetheless very challenging. In fact, accurate measurements would require a high quality model of the cockpit structure not only in layout, but also in structural response, or purposely-designed actuation systems able to reproduce the vibrations of the displays. Costs associated with such a solution may only be justified later in the design process when choices are established, and the room for introducing modifications thin. Therefore, during the early phases of rotorcraft design, when extensive modifications are still possible, reliance on numerical analysis is mandatory. This is also true when retrofitting an existing design.

A multidisciplinary simulation environment favoring modularity is required to develop accurate and computationally efficient models of the coupled rotorcraft-pilot dynamics. The combined model is thus made of the assembly of sub-components, representing different dynamics: the rotor aeroelastic behavior, governing the vibratory load generation and initial path of transmission; the structural model of the fuselage and cockpit, detailing the transmission of vibrations to the cockpit display; finally, the biomechanical model of the pilots and occupants, involving the propagation of vibrations through the body.

MASST (Modern Aeroservoelastic State Space Tools), a tool developed at Politecnico di Milano, is designed to handle such duty. It analyzes compact, yet complete modular models of linearized aeroservoelastic systems [18, 21]. Rotorcraft subcomponents are collected from well-known, reliable and state-of-the-art sources. After being assembled using the Craig-Bampton Component Mode Synthesis (CMS) substructuring method for linear substructures [22], they are cast into state-space form. This approach is crucial to be able to model helicopter subcomponents in their most suitable environments and compose the overall model. The model assembled in MASST is cast into the classical state space form for Linear Time Invariant (LTI) systems, defined by the quadruple $\mathbf{A}, \mathbf{B}, \mathbf{C}, \mathbf{D}$ :

$\dot{\mathbf{x}}=\mathbf{A x}+\mathbf{B u}$

$\mathbf{y}=\mathbf{C x}+\mathbf{D u}$

where vector $\mathbf{x}$ contains the states of the system, $\mathbf{y}$ is the system output, and $\mathbf{u}$ includes the inputs.

When expressed in the Laplace domain, the input-output relationship

$\mathbf{y}(s)=\left[\mathbf{C}(s \mathbf{I}-\mathbf{A})^{-1} \mathbf{B}+\mathbf{D}\right] \mathbf{u}(s)=\mathbf{G}(s) \mathbf{u}(s)$. 
is obtained, where $\mathbf{G}(s)$ is the corresponding transfer matrix between inputs and outputs. The system matrices often result from the linearization of general problems about prescribed values of selected parameters. Models at intermediate parameter values can be obtained by consistent interpolation of the matrices.

Following the description presented above, MASST can adequately support rotorcraft ride quality assessment, a special case of which instrument reading ability is, since it

1. provides high-fidelity overall modeling through subcomponent assembly;

2. allows flexibility in the source of sub-component formulation;

3. enables the definition of sensor-force relations between arbitrary structural points;

4. exports proper models apt to the interaction with vibration devices and human biodynamic models. This eliminates the need to reassemble the whole model when a subsystem changes, thus making MASST an effective and thoroughly parametric simulation environment.

With regard to the above-mentioned features, MASST has proven its capabilities considering its efficient use in rotorcraft helicopter and tiltrotor aeroservoelasticity [18, 21], vibration analysis [23], and handling of biodynamic models of the human body with increasing and arbitrary levels of complexity [24].

\subsection{Visual vibration assessment}

A detailed vibratory field resulting from vibration sources can be obtained using a high fidelity vehicle model. Such a model is expected to include the acceleration of the instrument panel $\left(\mathbf{a}_{\text {panel }}\right)$ for the purpose of visual performance. In addition to that, the acceleration of the eye $\left(\mathbf{a}_{\text {eye }}\right)$ is also required for a complete assessment of visual acuity degradation. It is not expected that the two eyes vibrate in a significantly different way, therefore both eyes are assumed to have the same response. However, if there are two pilots this should be considered in the formulation. For the latter, the acceleration at a specific point of the panel (e.g., the panel center) can be considered or, alternatively, the average vibration of a set of points, or even all the points on the panel can be used. In this work, the panel vibration vector represents the average of the panel nodal accelerations, unless otherwise stated.

The acceleration of the eyes and of the points on the panel are vectors:

$\mathbf{a}_{\mathrm{eye}}=\left[\begin{array}{lll}a_{\mathrm{eye}, x} & a_{\mathrm{eye}, y} & a_{\mathrm{eye}, z}\end{array}\right]^{T}$ $\mathbf{a}_{\text {panel }}=\left[\begin{array}{lll}a_{\text {panel }, x} & a_{\text {panel }, y} & a_{\text {panel }, z}\end{array}\right]^{T}$

Among the three components of those accelerations, the vertical one, $a_{i, z}, i=$ eye,panel, is the most significant for the impact of vibrations on vision [4]; thus, it is considered the critical component also in the present work. Dropping the direction subscripts, the eye and panel acceleration becomes:

$\mathbf{a}_{\mathrm{eye}} \approx a_{\mathrm{eye}, z}=a_{\mathrm{eye}}$

$\mathbf{a}_{\text {panel }} \approx a_{\text {panel }, z}=a_{\text {panel }}$

The two accelerations result from the transmission of timedependent loads from the rotor, through the airframe and the cockpit and the pilots' bodies, respectively. The MASST model is linear: therefore, the magnitude of the relative acceleration between the eye and the panel can be considered as a measure of the severity of the impact of vibration on the pilot display reading ability. Considering the $i$ th pilot, a Visual Vibration Index (VVI) can be defined as follows:

$\mathrm{VVI}_{i, \text { sub }}=\left|a_{i, \text { ye }}-a_{i, \text { panel }}\right|$

where the subscript 'sub' refers to the difference between the two accelerations. According to Eq. 7, if eye and panel vibrate at the same frequency and amplitude, and in phase, the instrument reading ability is not affected. However, the estimate given by this parameter could be over-optimistic, also considering the wider margin of error associated with the estimation of the phase with respect to that of the amplitude. Therefore, a more conservative scenario can also be considered, assuming that the eye and the panel vibrations are always in phase opposition:

$\mathrm{VVI}_{i, \text { sum }}=\left|a_{i, \text { eye }}\right|+\left|a_{i, \text { panel }}\right|$

where the subscript 'sum' refers to the summation of the amplitudes of the two accelerations.

This formulation can be extended to cover the presence of a second pilot, which is quite common in helicopters. In this case, the overall VVI can be assumed to be the average of the two pilots' indices:

$\mathrm{VVI}_{\mathrm{av}}=\frac{\mathrm{VVI}_{1}+\mathrm{VVI}_{2}}{2}$

$\mathrm{VVI}_{i}$ being either $\mathrm{VVI}_{i, \text { sub }}$ or $\mathrm{VVI}_{i, \text { sum }}$. Alternatively, a conservative formulation would be achieved if the maximum VVI among those of the two pilots is considered:

$\mathrm{VVI}_{\max }=\max \left(\mathrm{VVI}_{1}, \mathrm{VVI}_{2}\right)$

The combination of these alternatives, Eqs. (7-10), leads to the four VVI definitions reported in Table 1. 
Table 1 Nominal and more conservative visual vibration index formulations

\begin{tabular}{lll}
\hline & Average of Two pilots & $\begin{array}{l}\text { Maximum } \\
\text { of two } \\
\text { pilots }\end{array}$ \\
\hline $\begin{array}{l}\text { Norm of subtracted accel- } \\
\text { erations }\end{array}$ & $\mathrm{VVI}_{\text {sub,av }}$ & $\mathrm{VVI}_{\text {sub,max }}$ \\
$\begin{array}{l}\text { Sum of acceleration norms } \\
\mathrm{VVI}_{\text {sum,av }}\end{array}$ & $\mathrm{VVI}_{\text {sum,max }}$ \\
\hline
\end{tabular}

These four combinations can be considered the most plausible formulations. Given the high fidelity modeling capability of MASST, the VVI formulation can be expanded to more complex forms by adding an arbitrary number of vibratory loads and acceleration sensors. The level of detail used in modeling the sub-components is the sole limiting factor.

\section{Analysis model}

The methods described in the previous section allow us to build a complete, modular, high-fidelity model of the helicopter and human occupant dynamics that is able to predict the effect of aircraft vibrations on the visual performance analysis. The accuracy of the predicted vibrations depends on the availabily of detailed data. In the present work, the proposed approach is illustrated using available data related to a generic helicopter configuration, without claiming any strict correlation with experimental data, for illustrative purposes. It is however worth stressing that the situation corresponds to that of rotorcraft designers during the early stages of a new design when the level of detail of available data increases as the design is refined, but experimental data are not yet available.

\subsection{Aeroservoelastic helicopter model}

A state-space model of the helicopter structural dynamics is at the core of the complete model used in the analysis. Accurate modeling of the rotorcraft loads that excite the structure vibrations, and the correct assessment of the vibration propagation paths is of paramount importance in evaluating Noise, Vibration and Harshness (NVH) aspects.

The model used in the analysis is representative of a five blade, soft in-plane main rotor, medium weight helicopter (Fig. 1). It includes several aspects of the aircraft dynamics, which will be briefly described in the following paragraphs.

Flight Mechanics. The airframe six degrees of freedom dynamics is augmented with its stability derivatives, computed through look-up tables of the fuselage, horizontal
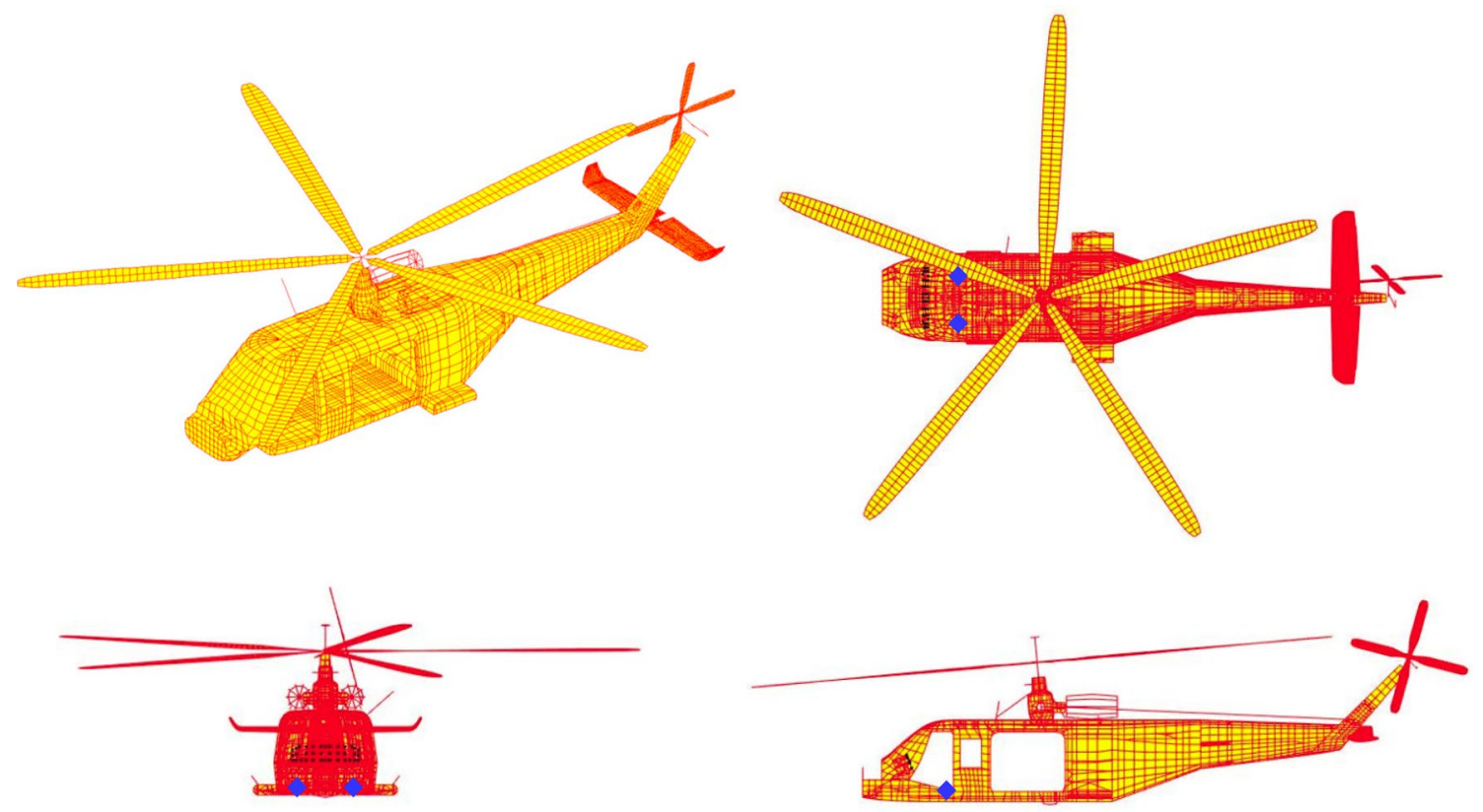

Panel Nodes

Fig. 1 Snapshot of the airframe and rotors used in the analysis 
Table 2 Numerical values for the seat-cushion model

\begin{tabular}{llll}
\hline & $m_{i}(\mathrm{~kg})$ & $c_{i}\left(\mathrm{NS} \mathrm{m}^{-1}\right)$ & $k_{i}\left(\mathrm{kN} \mathrm{m}^{-1}\right)$ \\
\hline Seat & $13.5^{1}$ & $750.00^{1}$ & $22.6^{1}$ \\
Cushion & $1.0^{2}$ & $159.00^{1}$ & $37.7^{1}$ \\
\hline
\end{tabular}

${ }^{1}$ From Ref. [27]; ${ }^{2}$ assumed

tail surface, and vertical empennage aerodynamics in CAMRAD/JA[25].

Aeroelasticity. From a detailed NASTRAN model, comprising more than 30000 nodes and 17000 elements (beam, shells, solids), the normal vibration modes of the airframe in the frequency band up to $50 \mathrm{~Hz}$, i.e. about twice the blade passage frequency, are extracted. The main and tail rotor models are formulated in CAMRAD/JA, and include the first lead-lag and the second flapping bending modes, as is typical for soft in-plane articulated rotors, and a torsion mode associated with the compliance of the control chain. A structural damping factor of $1.5 \%$ is assumed throughout. The rotor models are formulated in multiblade coordinates. The main rotor model is also completed by a Pitt-Peters [26] axial inflow model, with one state.

Control. The simplified dynamics of the blage pitch control servoactuators is modeled in Matlab/Simulink, including the effects of dynamic compliance and the coupled hydro-mechanical behavior of the servo-valve. Linear displacements are converted into collective and cyclic commands by considering the corresponding gear ratio coefficients.

Sensors. Virtual accelerometers are placed in the pilot seat locations, and the instrument display panels.

\subsection{Floor-human interface}

The interface between the pilot and the helicopter is modeled through an adaptation of the simplified seat and cushion model presented in [27]. Lumped masses associated with the cushion and the seat are suspended through a spring and damper system, and connect to the pilot model at the buttocks. The model is sketched in Fig. 2, with data given in Table 2.

\subsection{Upper body biodynamics model}

Human biodynamics is predominantly modeled using three approaches:

- (Linear) Lumped-Parameter Modeling (LPM), making use of point masses, ideal spring and damper elements;

- Finite element Method (FEM);

- Multibody Dynamics (MBD)

All three approaches can be (and have been) used for comfort assessment; however, FEM and MBD are more capable than LPM when upper body segments are of interest [24]. Therefore, the present work utilizes a multibody dynamics model to better estimate the acceleration of the head. A sitting human, the typical posture of helicopter pilots, is considered.

$\operatorname{MBDyn}^{1}$ [28], a free, general-purpose multibody solver, was used in developing the MBD model of the upper body. As presented in Fig. 3, the MBD model comprises 34 rigid bodies, associated with the sections of the trunk corresponding to each vertebra from $\mathrm{C} 1$ to $\mathrm{S} 1$, and 8 visceral masses. Relative vertebral displacements are assumed to lie
Fig. 2 Lumped parameter seat and cushion model [24]

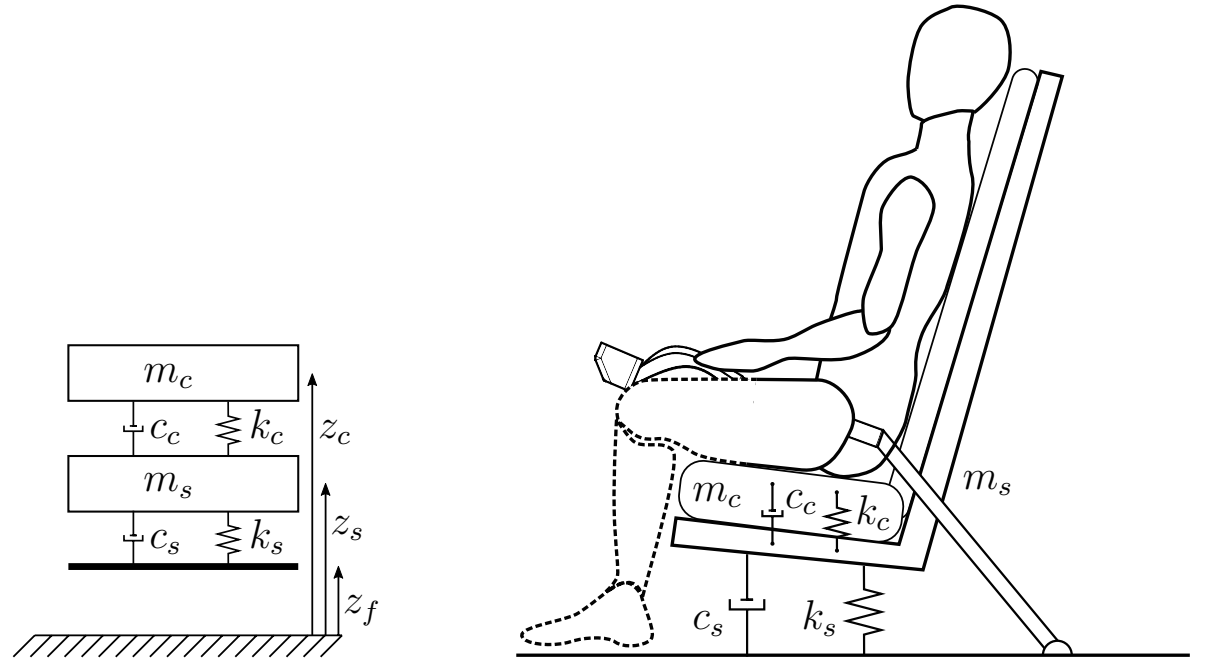

$\overline{1}$ http://www.mbdyn.org/, last accessed March 2021. 
Fig. 3 Multibody model of a sitting human [24]

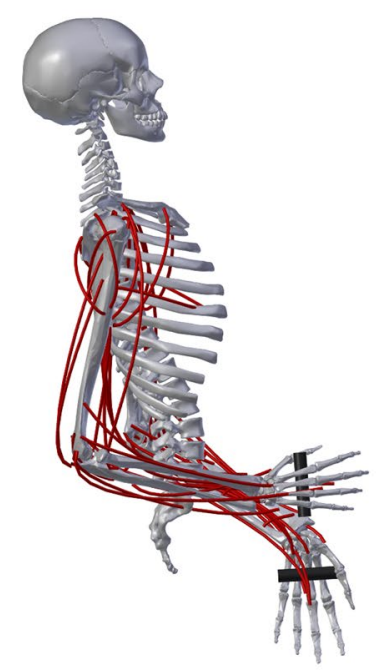

in the direction locally tangent to the spine axis. The relative motion of the vertebrae along the anatomical anteroposterior and medio-lateral directions is constrained. Linear viscoelastic elements, acting on all the unconstrained degrees of freedom, connect the vertebrae. Visceral masses have non-negligible inertia, and their dynamics is excited through a different load-path with respect to vertebral sections, hence they can significantly affect the vibration dynamics of the upper body [29]. For this reason, they are also connected to the corresponding vertebrae, from $\mathrm{T} 11$ to $\mathrm{S} 1$, and between them, through linear viscoelastic elements. Additional lumped masses, representing the upper limbs, the head and a third of the mass of the thighs are respectively placed in correspondence to the centers of the shoulder girdles, of the head and of the pelvis. The pelvic area modeling is completed by the introduction of a mass and a viscoelastic element representing the buttocks, through which the body contacts the seat surface. The motion of the buttocks is constrained as to allow only the vertical relative displacement with respect to the S1 vertebra and the rotations in the sagittal plane (i.e. the anatomical plane ideally dividing the human body into left and right portions) and coronal plane (i.e. the anatomical plane ideally dividing the human body into anterior and posterior portions).

In the MBD human biodynamic model, the structural properties of the building blocks of the body can be used to construct the overall model. However, the mechanical properties of these building blocks vary within a population; hence, a statistical parametrization is needed to obtain practical ranges for the parameter values. The required mechanical properties of the human body parts (muscles, bones, etc.) are usually obtained through cadaver dissection and analysis [30, 31]. An alternative is to use non-invasive measurements and imaging techniques to gather information in vivo $[32,33]$. In this work, values
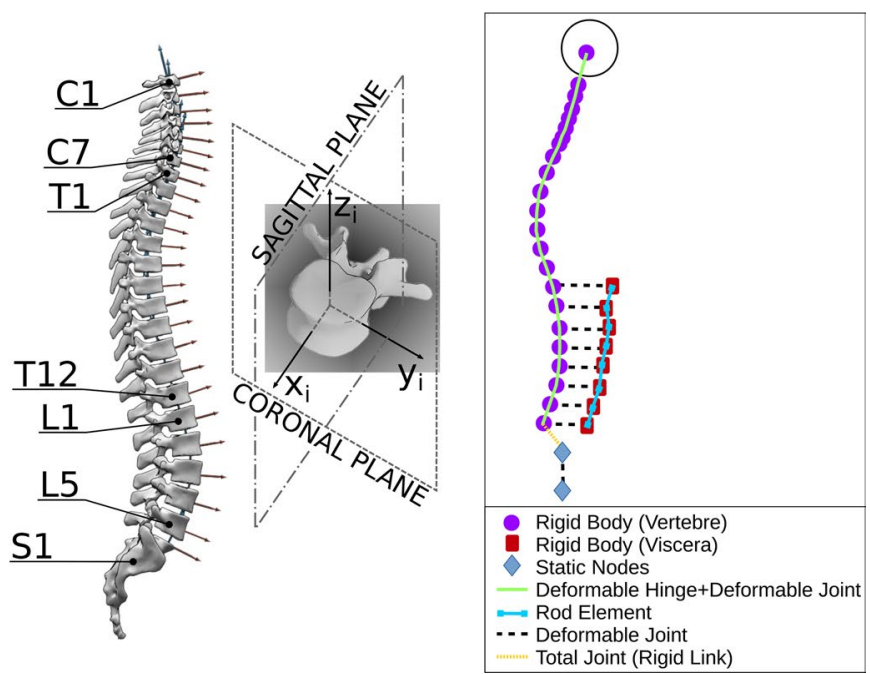

of the intervertebral and vertebra-viscera stiffness coefficients in the sagittal plane are taken from Ref. [34] and the reference values for stiffness and damping coefficients for intervertebral elements in the other directions are obtained from Ref. [35]. For elements connecting viscerae to vertebrae and viscerae to viscerae, the damping is assumed to be directly proportional to the stiffness, with a coefficient of 0.1 , as reported in Ref. [34].

The topology of the multibody model is generated referring to the parametric ribcage geometry published by Shi et al. [36]. The ribcage model has been used to identify the most likely anthropometric parameters of the Kitazaki and Griffin [34] model. A 34 years old male, $1.78 \mathrm{~mm}$ tall weighting $84 \mathrm{~kg}$, for a body mass index (BMI) of approximately 26.5 was found through an optimization procedure.

The corresponding estimated ribcage dimensions are compared with the one of the reference subject using scaling factors along the three dimensions $\lambda_{x}, \lambda_{y}, \lambda_{z}$. They are subsequently used to estimate the variation of the model geometry with respect to the reference one: the initial position of the nodes and the initial configuration of the algebraic constraints is obtained by directly scaling the related position vectors components with the $\lambda_{i}$ coefficients. The masses are scaled keeping the ratio between the individual masses constant, ensuring that the mass of the trunk accounts for $68 \%$ of the total body mass, and that the mass of the viscerae constitutes $20 \%$ of the total body mass [37].

Intervertebral elements' stiffness and damping coefficients are scaled using the following procedure:

1. the ratio between each stiffness or damping coefficient and the corresponding mass element is evaluated on the reference parameters of [34];

2. the resulting ratios are multiplied by the corresponding scaled masses; 
Fig. 4 Eye response to unit head acceleration. Plot based on data from Ref. [13]

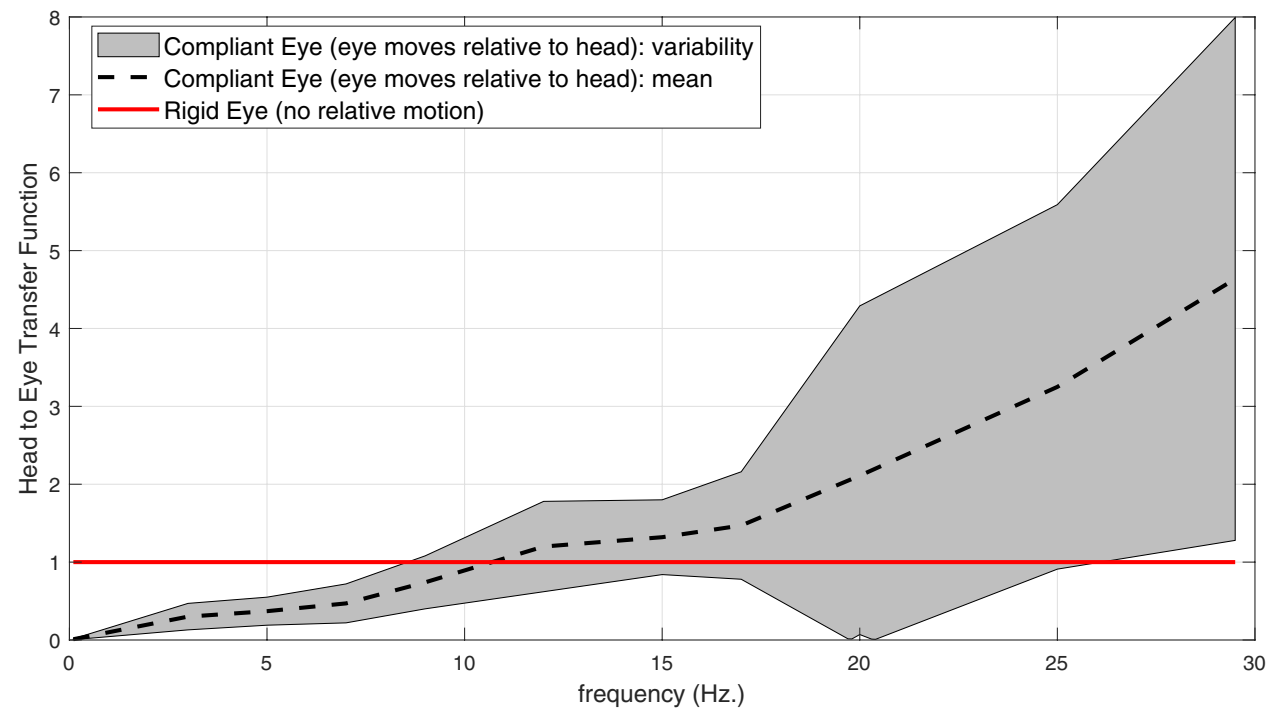

3. the resulting stiffness coefficients are modified by the value of a second-order polynomial function of the BMI of the subject, $\mathcal{P}(\mathrm{BMI})$.

Taking as an example the $i$-th stiffness coefficient $K_{i}$, the scaling relationship is:

$K_{i}=\mathcal{P}(\mathrm{BMI}) \frac{k_{i}}{m_{i}} M_{i}$

where $K_{i}, M_{i}$ indicate the scaled coefficients, $k_{i}, m_{i}$ the reference ones, and $\mathcal{P}(\mathrm{BMI})$ the correction polynomial obtained by fitting the model response, in terms of the first vertical resonance frequency of the spine, to experimental data. Readers may refer to Ref. [24, 38] for more details of the upper body model.

\subsection{Ocular dynamics}

The MBD model explained above represents the human dynamics from the buttock, where the vibrations enter the body and propagate to the head. Together with the seatcushion model, the acceleration at the head as a result of the acceleration at the cabin floor can be estimated. From the head, however, vibrations are further transferred to the eye. As a result, the eye moves at an amplitude and phase that differs from that of the head, therefore spoiling the image of the display on the retina. This behaviour of the eye can be modeled using the same computational techniques that are used in biodynamic modeling (see for example Ref. [39]). However, a simpler approach, based on experimental transfer functions, may be preferred, since:
1. the mass of the eye is very small with respect to that of the head; therefore, the coupling between head and eye motion is negligible;

2. the head and torso can induce great variability in the response, considering changes in posture and seat inclination, while the eye's dynamics is much less affected by the change in orientation;

3. a detailed modeling of the eye would require a very complex, multiphysical and multidomain analysis, which is not mature at this point.

Considering the above-mentioned issues, rather than building a complete physical model of the eye or completely ignoring its effects, an empirical transfer function of the eye identified from experiments was preferred, to include the ocular dynamics and investigate its effects. As mentioned in Sect. 1, experiments achieved similar trends regarding the dynamic behaviour of the eye at high frequencies. Among the above-cited studies, Ref. [13] is preferred, since the head to eye transfer function including magnitude, phase and their standard deviation were provided. The reported dynamics are shown in Fig. 4 with its mean value and standard deviation of the subject group. The passive, or compliant, eye motion is also included for reference. A general trend can be observed, though the variation increases with increased frequency.

When the head to eye transfer function is multiplied by the head acceleration obtained using the biodynamic model, the estimated acceleration of the eye is obtained. Once the frequency-dependent response of the eye is obtained, it can then be used in visual vibration index formulations. In this work, the mean value of the eye response is preferred when 


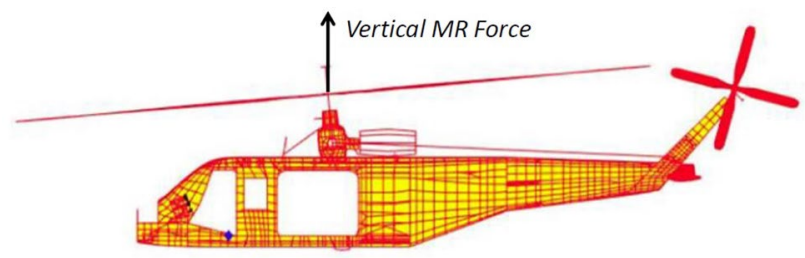

Fig. 5 Vertical force acting on the main rotor (MR) hub

the effect of other parameters is addressed, while the limits are used when the variability of the eye response is analyzed.

\subsection{The vibration source}

The vibration sources acting on the helicopter directly affect the acceleration measures at the pilots and display surface locations. MASST, the proposed simulation environment, allows to consider an increasing number of vibration sources. However, the vibration sources are limited to rotors, transmission, engines and rotor-fuselage interaction when a typical operation is considered. Among these, the major and persistent source of vibratory loads is the main rotor, while the rest contribute partially and occasionally [40]. The main rotor loads include the three force and moment components originated from each blade and summed at the main rotor hub, among which the vertical (i.e. in the direction of gravity) force is typically much larger in magnitude [41]. Therefore, the vertical hub force is selected as the vibration source, as visualized in Fig. 5. The forcing is applied as a unit force of varying frequency. In this case, a transfer function is obtained, which is a property of the system, independent of the forcing amplitude. In this work, transfer functions are used to analyze the effect of input frequency change on visual acuity and instrument reading ability degradation.

\section{Results and discussion}

This section presents how the proposed high-fidelity helicopter model can help to assess visual vibration degradation. The analyses are made in the $1 \mathrm{~Hz}-30 \mathrm{~Hz}$ frequency range. The lower limit is defined taking into account the cognitive correction of vibration at low frequencies as explained in Sect. 1. The upper limit is defined based on typical excitation frequencies of the main rotor loads [40], which typically reduce in amplitude with increasing frequency. As anticipated, in forward flight excitation may occur at the frequency corresponding to the angular velocity of the rotor, $\Omega$, in case of blade unbalance, or at the blade passage frequency, $N_{b} \Omega$. As reported in Table 1 of [20], $\Omega$ may vary from about $2 \mathrm{~Hz}$ to $8 \mathrm{~Hz}$, with values for typical medium weight helicopters in the $4 \mathrm{~Hz}-6 \mathrm{~Hz}$ range. Correspondingly, $N_{b} \Omega$ may vary from about $10 \mathrm{~Hz}$ to $30 \mathrm{~Hz}$, with a vast majority of helicopters in the interval $20 \mathrm{~Hz}-25 \mathrm{~Hz}$. For example, the data used in the present work refer to $\Omega \approx 5 \mathrm{~Hz}$ and $N_{b}=5$, i.e. $N_{b} \Omega \approx 25 \mathrm{~Hz}$.

First of all, the alternative VVIs defined in Table 1 are compared, to select the most apt formulation, as shown in Fig. 6. The nominal VVI, i.e. subtracted accelerations and average of pilots, has the lowest VVI, as expected. Generally, all formulations follow a similar trend, with VVI estimates of the same order of magnitude. There are non-negligible differences, though, a significant one occurring at very low frequencies near $2 \mathrm{~Hz}$, where the cognitive correction is involved in the ocular dynamics. Another difference can be observed between 23 and $26 \mathrm{~Hz}$. VVI sum,max $_{\text {max }}$, in which the amplitudes of the eye and panel accelerations are summed for the two pilots, is used as the baseline in the rest of the paper, as it provides significant margins of safety at the mentioned frequency intervals.
Fig. 6 Alternative visual vibration index formulations (VVI)

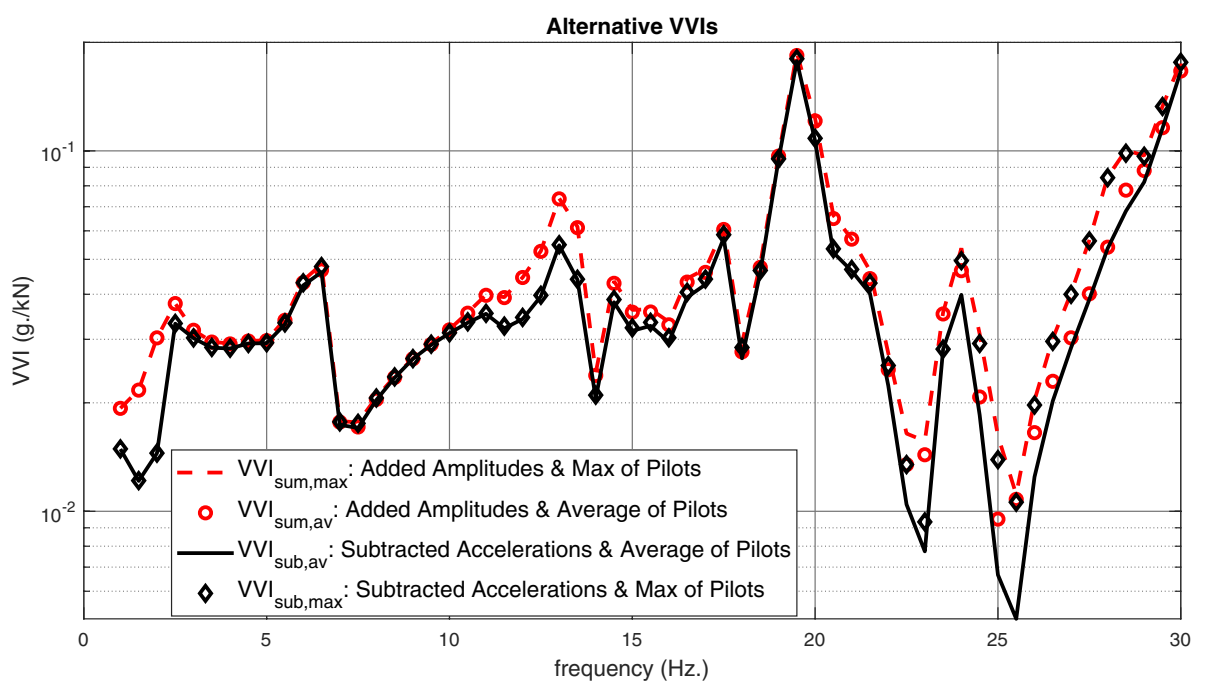


Fig. 7 Contribution of pilot and panel vibration on visual vibration index (VVI)
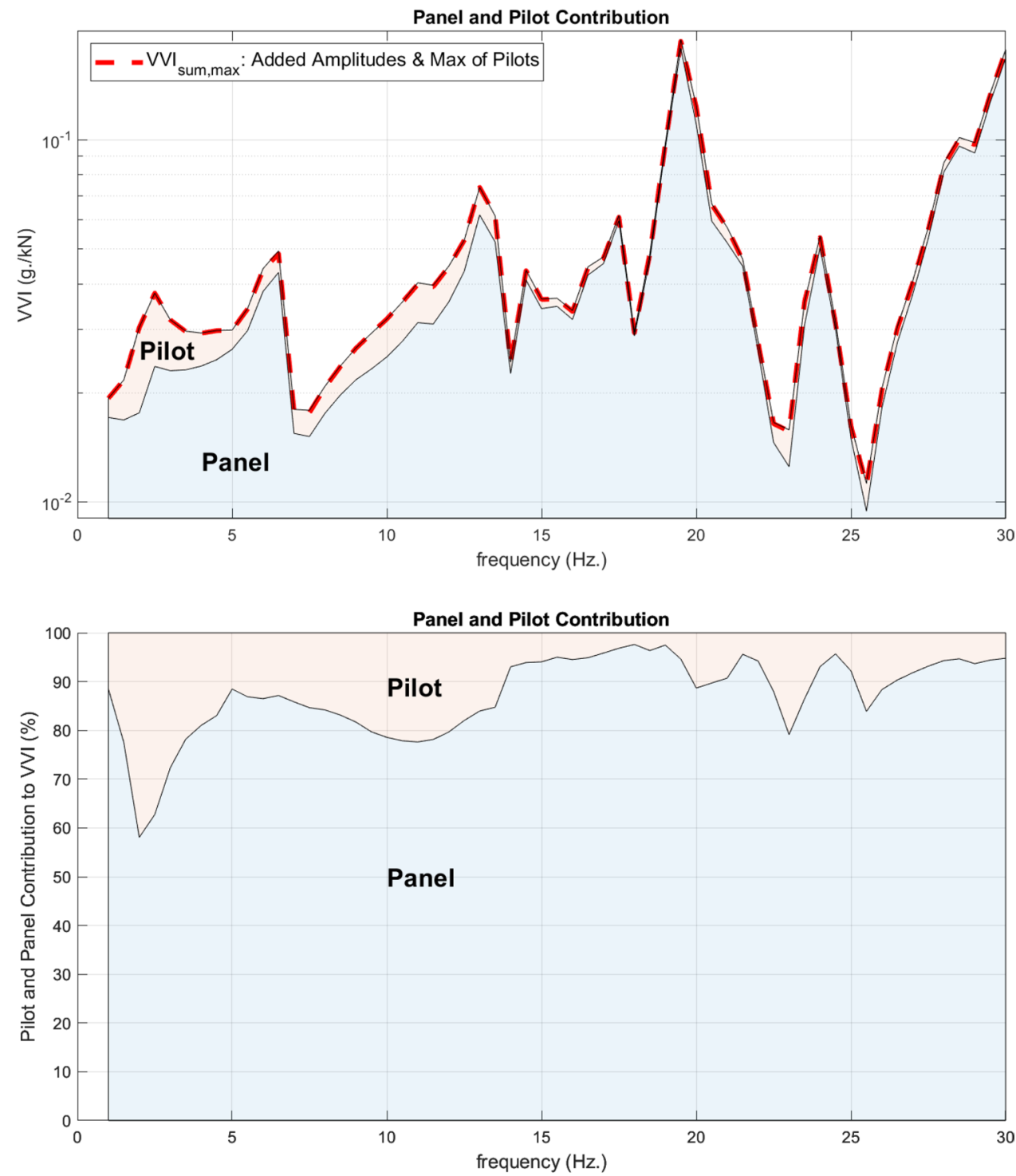

For the selected formulation ( $\left.\mathrm{VVI}_{\text {sum,max }}\right)$, the relative contributions of pilot and panel can be estimated by separating their respective accelerations that contribute to the $\mathrm{VVI}_{\text {sum,max }}$. As can be seen in Fig. 7, the area under the $\mathrm{VVI}_{\text {sum,max }}$ curve is separated into two regions. The bottom area shows the panel contribution, whereas the upper region presents the pilot contribution. It can be observed that the panel accelerations dominate the vibration index. The pilot contribution is significant under $14 \mathrm{~Hz}$, having the largest contribution around $3 \mathrm{~Hz}$. Over $14 \mathrm{~Hz}$, the pilot contribution to the $\mathrm{VVI}_{\text {sum,max }}$ is negligible and can be safely neglected.

Since the panel appears to dominate the response, it is worth evaluating the variability induced by the panel acceleration field. Being a wide area for two pilots, the panel vibration field may not be distributed evenly. For this purpose, the above-selected formulation $\left(\mathrm{VVI}_{\text {sum,max }}\right)$ was recalculated for the maximum and minimum acceleration on the panel grid. The result is the shaded area presented in Fig. 8. It can be observed that the variability in VVI due to panel acceleration distribution increases with the frequency. However, it can also be stated that the increase in VVI results in a value that is within the same order of magnitude of the nominal one.

As mentioned in the previous Section, and visualized in Fig. 4, the head to eye transfer function shows some variability. The formulations compared in Fig. 6 used the mean value of the head to eye response. Therefore, the effect of this variability on VVI is of interest, which can be depicted in Fig. 9. The line in the middle of the area belongs to the $\mathrm{VVI}_{\text {sum,max }}$ using the mean value of eye response. The area surrounding this line is shaded based on the minimum and maximum values of eye response, 
Fig. 8 Effect of panel vibration variability on visual vibration index (VVI)
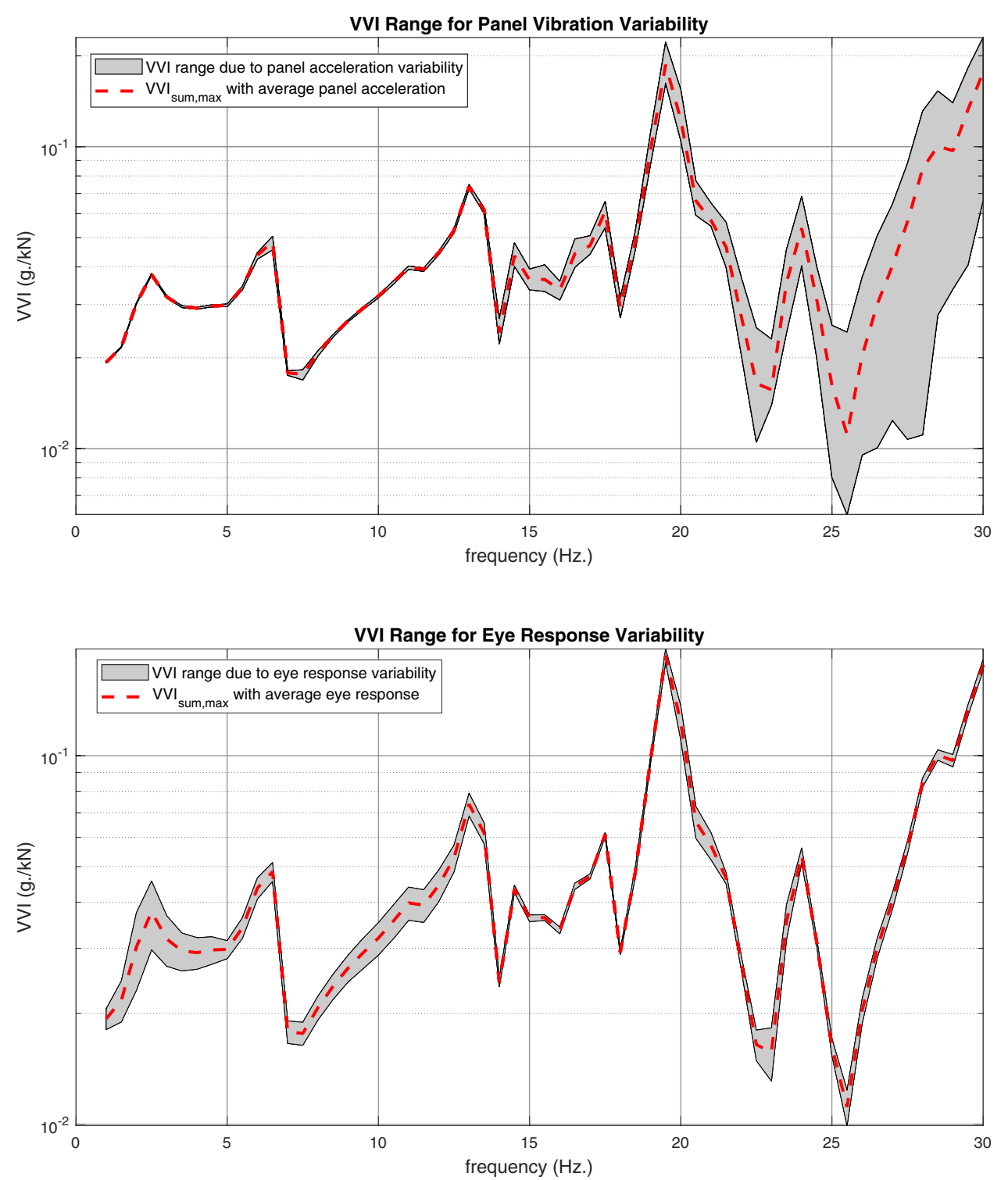

Fig. 9 Effect of eye response variability on visual vibration index (VVI) therefore providing insight on the effect of eye variability on VVI. It can be observed that the variability is greatest under $7 \mathrm{~Hz}$. This an interesting result as the eye response variability is lower in that region as compared to higher frequencies. Therefore, a physical modeling or experimental identification of the eye can prioritize frequencies under this threshold rather than higher frequencies, to reduce the variability in VVI estimates.

The dominance of panel vibration, especially at higher frequencies like the blade passing ones, suggests that reducing the vibrations of the panel, e.g. through passive means, would be beneficial. On the other hand, the significant contribution of the eye at frequencies near the rotor angular speed, which occurs when the blades are unbalanced, could be alleviated by either minimizing the rotor unbalance or by better insulating the pilot's seat.
A benefit of using a modular simulation environment is the ability to assemble and disassemble the sub-modules of rotorcraft components easily. This makes observing the contribution added by rotorcraft components possible, which can help identify the components that contribute most to the degradation in visual acuity and instrument reading ability. The detailed fully coupled model developed in the previous section is used as a baseline to analyze the relative importance of different parameters. With respect to the baseline overall model, the key elements of visual performance degradation are replaced with their simpler versions one at a time to address the sensitivity to modeling complexity. For each element, the full and reduced models are compared in the same frequency range $1-30 \mathrm{~Hz}$. A relative change in the VVI with respect to the $\mathrm{VVI}_{\text {sum,max }}$ (as reference, $\mathrm{VVI}_{0}$ ) of Fig. 6 is plotted in Fig. 10. 
Fig. 10 Relative change in visual vibration index (VVI)
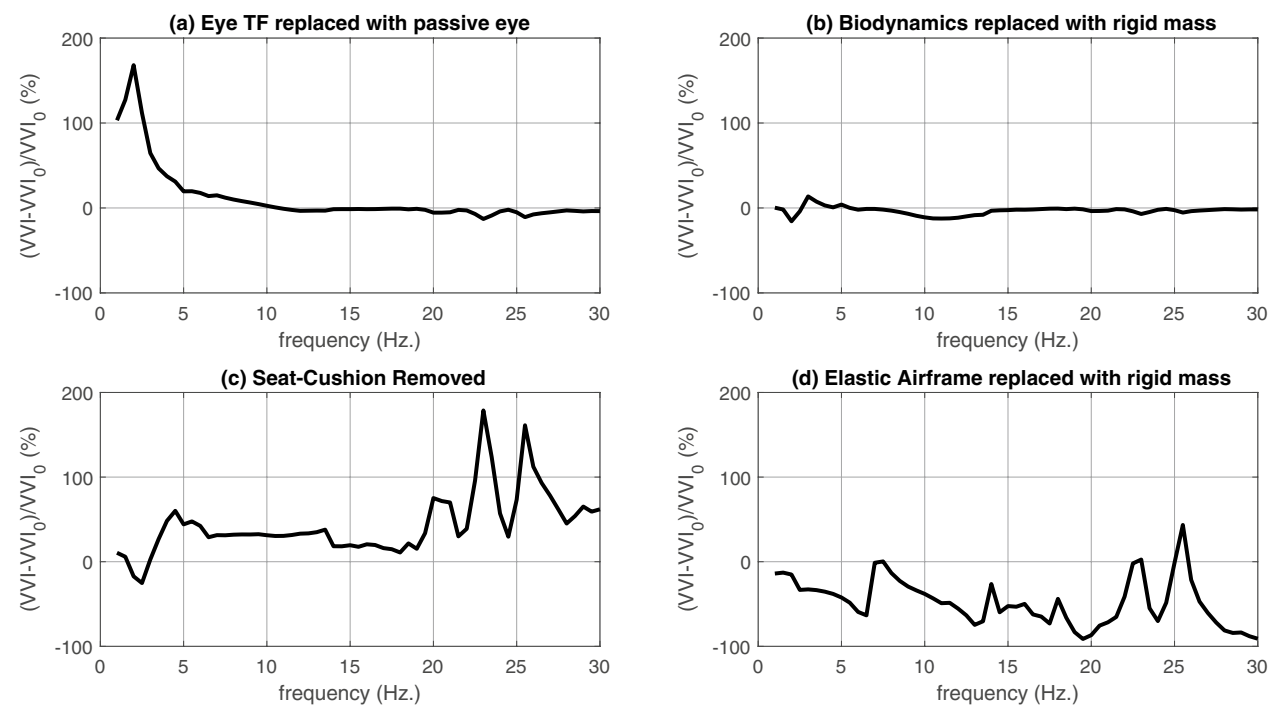

Four contributions in the load path from the main rotor vibratory loads to the motion of the eye are considered: (a) eye dynamics, (b) pilot biodynamics (c) seat-cushion compliance and (d) airframe structural dynamics.

(a) The first parameter is the eye dynamics. The transfer function representing the response of the eye is removed from the system and the vibration of the eye is assumed to be equal to that of head, i.e. the eye passively follows the head. The most significant differences occur at frequencies lower than $7 \mathrm{~Hz}$, and the inclusion of the eye transfer function eliminates overestimation of VVI. In other words, under $7 \mathrm{~Hz}$, the eyes dynamics dampen the vibrations acting on it. In the rest of the plot, there is no remarkable effect of the local eye motion. Therefore excluding the dynamics of the eye can be considered a conservative approach.

(b) The Second parameter is the pilot biodynamics, whose effects are evaluated by substituting the complete multibody model of the pilot's upper body with an equivalent rigid mass. It can be observed that there are local increases in VVI, below $20 \%$.

(c) The effect of the seat-cushion subsystem dynamics on the VVI is the second largest contributor after helicopter aeroelasticity; a significant change occurs over the whole frequency range. Similar to the dynamics of the eye, if the seat-cushion is not included, the VVI estimation becomes conservative.

(d) Among the selected elements, the most substantial discrepancy occurs when the aeroelastic model of the helicopter is replaced with a rigid body of equal mass. The significant change in VVI shows that the major contribution to VVI comes from the airframe poles and zeros, which suggests that the use of a high-fidelity aeroelastic model is essential. Although a considerable deformabil- ity is a consequence of light-weight aviation structures, the design can be optimized such that large VVI values caused by amplification at specific excitation frequencies are prevented. Examples of critical frequencies are the rotor angular speed, $\Omega$, and the blade passing frequency, $N_{b} \Omega$, where $N_{b}$ is the number of blades, respectively referred to as $1 / \mathrm{rev}$ and $N_{b} /$ rev in rotorcraft jargon. An alternative to modifying the poles of the aeroelastic system is the use of vibration attenuation devices, which can help achieving a safe separation of the dominant excitation frequencies and airframe poles.

\section{Conclusions}

In this work, a strategy to assess visual degradation under vibration exposure is presented, which can help rotorcraft designers to estimate the effect of visual performance under vibration exposure. A modular rotorcraft simulation environment is used as the foundations of the strategy. The reasons why such a tool is necessary for the mentioned purposes, and how such a model should be developed, have been discussed in detail. The vibration characteristics of a medium lift category helicopter is used. The load path from main rotor, the vibration source, to the eye and the instrument panels are described. Considering vertical vibrations of the panel and the eye, a visual vibration index is defined, hence the most critical components regarding the visual response is represented in the formulation.

The benefits of the suggested method are demonstrated using a high-fidelity simulation model, which includes an aeroelastic helicopter model, a detailed biodynamics model of the pilot, a dynamical model of the seat-cushion, and experimental results of ocular dynamics. From the analysis, the following conclusions can be drawn: 
1. the ocular dynamics - namely the ability to compensate head vibrations by moving the eyes-is very effective and dominates up to $5 \mathrm{~Hz}$, while its effect becomes negligible at higher frequencies;

2. the pilot biodynamics show a non-negligible contribution only in the low-frequency range;

3. over the target frequency range $(1 \mathrm{~Hz}-30 \mathrm{~Hz})$, the panel vibration dominates, while the pilot contribution to the visual vibration index is significant at relatively lower frequencies, especially around $5 \mathrm{~Hz}$. Better panel and/ or pilot insulation, depending on the frequency range of the main excitation, would be beneficial;

4. at high frequencies, the acceleration magnitude variability over the instrument panel can significantly change the visual vibration index as compared to the value estimated using the average acceleration;

5. the variability caused by the eye response is highest under $7 \mathrm{~Hz}$, while sensitivity reduces at higher frequencies. This suggest to aim at frequencies below this threshold in modeling or experimental identification of involuntary eye motion due to vibration for visual vibration assessment in helicopters;

6. the major contributor is the helicopter structural dynamics, as it is the common interface between the vibration source and the subjects, namely the pilots and instrument panel;

7. the seat-cushion dynamics can significantly affect the vibrations received by the pilots, therefore, causing amplifications of the vibration on the pilot over the whole frequency range. Considering that it is costly or sometimes even impossible to attenuate the dynamic response of the airframe, the seat-cushion becomes the most plausible candidate for mitigating the adverse effects of vibration on visual performance;

8. the numerical results presented the accelerations of the eye along the vertical direction, which is usually considered the most critical for display reading performance. The response of the eye along with the other directions could contribute as well; however, this requires further research, including the development of proper models and experimental investigations of the ocular dynamics along with the lateral and longitudinal directions.

Acknowledgements This work received partial support from Leonardo Helicopter Division. The authors particularly acknowledge LHD for providing part of the data used in the analysis.

Open Access This article is licensed under a Creative Commons Attribution 4.0 International License, which permits use, sharing, adaptation, distribution and reproduction in any medium or format, as long as you give appropriate credit to the original author(s) and the source, provide a link to the Creative Commons licence, and indicate if changes were made. The images or other third party material in this article are included in the article's Creative Commons licence, unless indicated otherwise in a credit line to the material. If material is not included in the article's Creative Commons licence and your intended use is not permitted by statutory regulation or exceeds the permitted use, you will need to obtain permission directly from the copyright holder. To view a copy of this licence, visit http://creativecommons.org/licenses/by/4.0/.

\section{References}

1. Masarati, P., Quaranta, G., Gennaretti, M., Serafini, J.: An investigation of aeroelastic rotorcraft-pilot interaction. In: 37th European Rotorcraft Forum, Gallarate, Italy, (2011). Paper no. 112

2. Quaranta, G., Tamer, A., Muscarello, V., Masarati, P., Gennaretti, M., Serafini, J., Colella, M.M.: Rotorcraft aeroelastic stability using robust analysis. CEAS Aeronaut. J. 5, 29-39 (2014). https://doi.org/10.1007/s13272-013-0082-z

3. Harrer, K.L., Yniguez, D., Maria, M.M. Ellenbecker, D., Estrada, N., Geiger, M.: Whole body vibration exposure for MH-60s pilots, In: 43th SAFE, Utah, USA, (2005)

4. Griffin, M.J.: Eye motion during whole-body vertical vibration. Hum Factors 18, 601-606 (1976). https://doi.org/10.1177/00187 2087601800608

5. Echeverria, D., Barnes, V., Bittner, A., Durbin, N., Fawcett-Long, J., Moore, C., Slavich, A., Terrill, B., Westra, C., Wieringa, D., Wilson, R., Draper, D., Morisseau, D.: Vision: The effects of vibration. The impact of environmental conditions on human performance. NUREG/CR5680, Division of Systems Research, Office of Nuclear Regulatory Research, U.S. Nuclear Regulatory Commission, (1994)

6. Gardner, C.: British Aircraft Corporation: A History by Charles Gardner, (1981)

7. Nakashima, A., Cheung, B.: The effects of Vibration Frequencies on Physical, Perceptual and Cognitive Performance. Technical Report DRDC Toronto TR 2006-218, DRDC Toronto (2006)

8. O'Briant, C.R., Ohlbaum, M.K.: Visual acuity decrements associated with whole body $\pm \mathrm{gz}$ vibration stress. Aerospace Med. 41, 79-82 (1970)

9. Ohlbaum, N.K.: Mechanical Resonant Frequency of the Human Eye in Vivo, Ph.D. thesis, Aerospace Mdical Research Laboratory, (1976)

10. Ishitake, T., Ando, H., Miyazaki, Y., Matoba, F.: Changes of visual performance indced by exposure to whole-body vibration. Kurume Med. J. 45, 59-62 (1998)

11. Collins, A.M.: Decrements in tracking and visual performance during vibration. Hum. Factors 15, 379-393 (1973). https://doi. org/10.1177/001872087301500409

12. Adelstein, B.D., Beutter, B.R., Kaiser, M.K., McCann, R.S., Stone, L.S., Anderson, M.R., Renema, F., Paloski, W.H.: Influence of Combined Whole-Body Vibration Plus G-Loading on Visual Performance. Technical Report NASA-TM-2009-215386, NASA, (2009)

13. Lee, R.A., King, A.I.: Visual vibration response. J. Appl. Physiol. 30, 281-286 (1971). https://doi.org/10.1152/jappl.1971.30.2.281

14. Lewis, C.H., Griffin, M.J.: Predicting the effects of vibration frequency and axis, and seating conditions on the reading of numeric displays. Ergonomics 23, 485-499 (1980). https://doi.org/10. 1080/00140138008924762

15. Harazin, B.: Louda, L., Pawlas, K., Jandak, Z.: Influence of whole-body vertical vibration on vision performance. Journal of Low Frequency Noise, Vibration and Active Control 15, (1996). https://doi.org/10.1177/026309239601500103

16. Clarke, N.P., Taub, H., Scherer, H.F., Temple, W.E., Vykukal, H.E., Matter, M.: Preliminary Study of Dial Reading Performance Duting Sustained Acceleration and Vibration, Technical Report AMRL-TR-65-110, AMRL, (1965) 
17. Tung, K., Millerand, M., Colombi, J., Uribe, D., Smith, S.: Effect of vibration on eye, head and helmet movements while wearing a helmet-mounted display. J. Soc. Information Display 22, 535-544 (2015). https://doi.org/10.1002/jsid.286

18. Masarati, P., Muscarello, V., Quaranta, G.: Linearized aeroservoelastic analysis of rotary-wing aircraft. In: 36th European Rotorcraft Forum, Paris, France, pp. 099.1-10 (2010)

19. Tamer, A., Zanoni, A., Cocco, A., Masarati, P.: Visual Performance Evaluation of Helicopter Pilots in Vibrating Cockpit. In: 15th International Conference on Multibody Systems, Nonlinear Dynamics, and Control, (2019). https://doi.org/10.1115/DETC2019-97525

20. Tamer, A., Zanoni, A., Cocco, A., Masarati, P.: A generalized index for the assessment of helicopter pilot vibration exposure. Vibration 4, 133-150 (2021). https://doi.org/10.3390/vibration4010012

21. Masarati, P., Muscarello, V., Quaranta, G., Locatelli, A., Mangone, D., Riviello, L., Viganò, L.: An integrated environment for helicopter aeroservoelastic analysis: the ground resonance case. In: 37th European Rotorcraft Forum, Gallarate, Italy, pp. 177.1-12 (2011)

22. Craig, R.R., Jr., Bampton, M.C.C.: Coupling of substructures for dynamic analysis. Journal 6, 1313-1319 (1968)

23. Tamer, A., Muscarello, V., Masarati, P., Quaranta, G.: Evaluation of vibration reduction devices for helicopter ride quality improvement. Aerospace Science and Technology 95, 105456 (2019). https://doi.org/10.1016/j.ast.2019.105456

24. Tamer, A., Zanoni, A., Muscarello, V., Cocco, A., Quaranta, G., Masarati, P.: Biodynamic modeling techniques for rotorcraft comfort evaluation. AMS 98, 147-158 (2019). https://doi.org/10.1007/ s42496-019-00014-5

25. Johnon, W.: CAMRAD/JA, A Comprehensive Analytical Model of Rotorcraft Aerodynamics and Dynamics, Johnson Aeronautic Version, organization. Johnson Aeronautics, (1988)

26. Pitt, D.M., Peters, D.A.: Theoretical prediction of dynamic-inflow derivatives. Vertica 5, 21-34 (1981)

27. Choi, Y.-T., Wereley, N.: Biodynamic response mitigation to shock loads using magnetorheological helicopter crew seat suspensions. J Aircraft 42, 1288-1295 (2005)

28. Masarati, P., Morandini, M., Mantegazza, P.: An efficient formulation for general-purpose multibody/multiphysics analysis. J. of Computational and Nonlinear Dynamics 9, (2014). https://doi.org/ 10.1115/1.4025628

29. Belytschko, T., Privitzer, E.: Refinement and Validation of a Three Dimensional Head-Spine Model. Technical Report Contract AF33615-76-C-0506. Air Force Systems Command, Wright-Patterson Air Force Base, Ohio (1978)

30. Pooni, J., Hukins, D., Harris, P., Hilton, R., Davies, K.: Comparison of the structure of human intervertebral discs in the cervical, thoracic and lumbar regions of the spine. Surg Radiol Anatomy 8, 175-182 (1986). https://doi.org/10.1007/BF02427846

31. Yoganandan, N., Kumaresan, S., Voo, L., Pintar, F., Larson, S.: Finite element modeling of the c4-c6 cervical spine unit. Med Eng Phys 18, 569-574 (1996). https://doi.org/10.1016/13504533(96)00013-6

32. Subrata, P. (ed.), Design of Artificial Human Joints \& Organs. Springer, (2014). https://doi.org/10.1007/978-1-4614-6255-2

33. Zheng, Y.-P., Mak, A.F.T.: ultrasound indentation system for biomechanical properties assessment of soft tissues in-vivo. IEEE Trans Biomed Eng 43, 912-918 (1996)

34. Kitazaki, S., Griffin, M.: A modal analysis of whole-body vertical vibration, using a finite element model of the human body. Journal of Sound and Vibration 200, 83-103 (1997) http://www.sciencedir ect.com/science/article/pii/S0022460X96906744. https://doi.org/ 10.1006/jsvi.1996.0674

35. Valentini, P.P., Pennestrì, E.: An improved three-dimensional multibody model of the human spine for vibrational investigations. Multibody Syst Dyn 36, 363-375 (2016). https://doi.org/ 10.1007/s11044-015-9475-6

36. Shi, X., Cao, L.: Reed, M., Rupp, J., Hoff, C., Hoff, C.., Hu, J.: A statistical human rib cage geometry model account for variations by age, sex, stature and body mass index. J Biomech $\mathbf{4 7}$, 2277-2285 (2014)

37. De Leva, P.: Adjustments to zatsiorsky-seluyanov's segment inertia parameters. J Biomech 29, 1223-1230

38. Zanoni, A., Cocco, A., Masarati, P.: Multibody dynamics analysis of the human upper body for rotorcraft-pilot interaction. Nonlinear Dynamics. Accepted for publication (2020). https://doi.org/10. 1007/s11071-020-06005-7

39. Pascolo, P., Carniel, R., Grimaz, S.: Dynamical models of the human eye and strabismus. Chaos, Solitons \& Fractals 41:24632470. https://doi.org/10.1016/j.chaos.2008.09.032.http://www. sciencedirect.com/science/article/pii/S096007790800427X

40. Johnson, W.: Rotorcraft Aeromech. Cambridge University Press, New York (2013)

41. Bramwell, A.R.S., Done, G., Balmford, D.: Bramwell's Helicopter Dynamics. Butterworth-Heinemann, Oxford (2001)

Publisher's Note Springer Nature remains neutral with regard to jurisdictional claims in published maps and institutional affiliations. 